dismissed by his senior colleague, Kinnear Wilson, who regards narcolepsy as a syndrome with several different causes, traumatic, endocrine, epileptic, toxi-infective, circulatory, tumor, and idiopathic. Neither Adie nor Kinnear Wilson refers to hyposmia in their account of narcolepsy-cataplexy disorder, but they did locate the pathology in the floor of the third ventricle in symptomatic cases. (Compston A. Brain Oct 2008;131:2532-2535).

\title{
ELECTROENCEPHALOGRAPHY
}

\section{PROGNOSTIC VALUE OF EEG IN ASPHYXIATED NEWBORNS TREATED WITH HYPOTHERMIA}

Researchers at Children's Hospitals in Milan, Italy, determined the prognostic value of electroencephalographic patterns in 23 newborns with severe perinatal hypoxic-ischemic encephalopathy, treated with hypothermia. EEG monitoring was obtained within 48 hours after birth, and at follow-up at ages 1 week, 1 month, 3-6 months, and 1 year. EEG background activity was classified as follows: 1) inactive pattern; 2) severe low-voltage continuous pattern; 3) trace alternant-like, discontinuous pattern; and 4) monomorphic middle-voltage, continuous $30-100 \mathrm{mcV}$ activity, with poor spatial and sleep-state organization. Pattern 1 (inactive) in the first 48 hrs was associated with death or severe neurologic sequelae. Pattern 2 (low-voltage continuous) at age 1 week indicated a poor prognosis, and the persistence of EEG abnormalities in $67 \%$ patients at age 1 month was associated with a higher risk of neurologic sequelae. A normal EEG at age 1 month was associated with a favorable outcome at age 1 year. After 1 month of age, the EEG is less sensitive but more specific in prediction of outcome, due to the natural trend toward normalization with age. At age 1 year, $52 \%$ infants had normal neurologic examinations, $13 \%$ had minor sequelae, and $17 \%$ major sequelae; $17 \%$ had died within 1 month of age. (Mariani E, Scelsa B, Pogliani L, Introvini P, Lista G. Prognostic value of electroencephalograms in asphyxiated newborns treated with hypothermia. Pediatr Neurol Nov 2008;39:317-324). (Respond: Dr Scelsa, Department of Child Neurology, Vittori Buzzi Children's Hospital, Via Castelvetro 32, 20154 Milan, Italy. E-mail: b.scelsa@icp.mi.it).

COMMENT. These results confirm previous findings that background EEG abnormalities detected in newborns soon after hypoxic-ischemic encephalopathy are predictive of outcome, even in patients treated with hypothermia.

\section{AMPLITUDE-INTEGRATED EEG IN THE NEWBORN}

Th value of amplitude-integrated electroencephalography (aEEG) in the newborn is explored by researchers at Washington University, St Louis; Wilhelmina Children's Hospital, Utrecht, Netherlands; and Uppsala University Hospital, Sweden. The system was originally designed to monitor lower amplitude signals of 1 to $10 \mathrm{mcV}$ and depressed cerebral activity in adults undergoing bypass surgery, as well as seizure activity. Meta-analysis has confirmed that the aEEG pattern in the first 6 hours of life of term newborns with hypoxic-ischemic encephalopathy is strongly predictive of outcome. Pattern-recognition may be more reliable than amplitude in the evaluation of aEEG. The electrode placement over parietal areas, 
coinciding with vulnerable watershed areas of cortex, yields a high sensitivity (81\%) for electrical seizure activity. Bedside aEEG monitoring "on-line" and around the clock is underway in the NICU to explore feasibility and impact on management of electrographic seizures. In contrast to a low sensitivity and high false-positive rate using electronic fetal heart monitoring, the early use of aEEG has a sensitivity of $85 \%$ to $91 \%$ for predicting neurodevelopmental outcome in term newborn infants with neonatal encephalopathy. aEEG is used to recruit patients for study of therapeutic hypothermia, and clinically to evaluate the newborn by complementing the neurologic examination, conventional EEG, and neuroimaging, not in isolation. (Shah DK, de Vries LS, Hellstrom-Westas L, Toet MC, Inder TE. Amplitude-integrated electroencephalography in the newborn: a valuable tool. Pediatrics Oct 2008;122:863-865). (Respond: Divyen K Shah MB ChB, Washington University, Department of Pediatrics, $8^{\text {th }}$ Floor, NW Tower, 1 Children's Place, St Louis, MO 63110. E-mail: shah-d@ kids.wustl.edu).

COMMENT. Analsis of clinical data at 18 months in a study of head cooling for neonatal encephalopathy found that infants with greater amplitude-integrated EEG, lower birth weight, absence of seizures, and higher Apgar score had significantly better outcomes. Gender and gestational age were not significantly associated with outcome. (Wyatt JS et al. Pediatrics 2007;119:912-921; Ped Neur Briefs June 2007;21:48).

\section{EEG IN SPECIFIC LANGUAGE IMPAIRMENT}

The value of routine wake electroencephalography in children with specific language impairment was reviewed retrospectively in 111 children examined over a 10-year interval at Montreal Children's Hospital, Quebec, Çanada. Children with known central nervous system disorders were excluded. Sleep-deprived EEG was not performed. The wake EEG was abnormal in $35(31.5 \%)$ children, including $7(6.3 \%)$ with epileptiform activity, and higher than that in 'normal' children $(3.54 \%)$. (NS. $\mathrm{P}=0.12)$. The epileptiform activity was active in 3 patients. Three $(2.7 \%)$ had excessive paroxysmal activity (PA) with hyperventilation, and 3 $(2.7 \%)$ had excessive PA with photic stimulation. Female gender had a small association with abnormal EEG. Two patients with epileptiform EEGs received anticonvulsant medication on parental request; subsequent EEGs but not the speech were improved. Soft neurologic signs, macrocephaly, or microcephaly were present in $21(18.9 \%)$ children, ADHD in $15(13.5 \%)$, and comorbid learning difficulties in $14(12.6 \%)$. (Venkateswaran S, Shevell $\mathrm{M}$. The case against routine electroencephalography in specific language impairment. Pediatrics Oct 2008;122:e911-e916). (Respond: Michael Shevell MDCM, FRCP(C), E-mail: Michael.shevell@muhc.mcgill.ca).

COMMENT. Wake EEG is of uncertain value in the routine diagnostic evaluation of children with specific language impairment. Definitive recommendations await further investigation with both wake and sleep EEG. A previous prospective study including both wake and sleep EEG demonstrated abnormalities in almost half of the patients (14 of 32) with developmental dysphasia, and epileptic activity in 30 of the 32 in overnight recordings. (Echenne B et al. Brain Dev 1992;14:216-225). Patients with receptive dysphasia were at highest risk for abnormal EEG. (Tuchman R et al. Pediatrics 1991;88:1219-1225). 